\title{
The Partial Second Boundary Value Problem of an Anisotropic Parabolic Equation
}

\author{
Huashui Zhan (D) \\ School of Applied Mathematics, Xiamen University of Technology, Xiamen, Fujian 361024, China \\ Correspondence should be addressed to Huashui Zhan; huashuizhan@163.com
}

Received 22 March 2019; Accepted 16 April 2019; Published 2 May 2019

Academic Editor: Maria Alessandra Ragusa

Copyright (c) 2019 Huashui Zhan. This is an open access article distributed under the Creative Commons Attribution License, which permits unrestricted use, distribution, and reproduction in any medium, provided the original work is properly cited.

Consider an anisotropic parabolic equation with the variable exponents $v_{t}=\sum_{i=1}^{n}\left(b_{i}(x, t)\left|v_{x_{i}}\right|^{p_{i}(x)-2} v_{x_{i}}\right)_{x_{i}}+f(v, x, t)$, where $b_{i}(x, t) \epsilon$ $C^{1}\left(\overline{Q_{T}}\right), p_{i}(x) \in C^{1}(\bar{\Omega}), p_{i}(x)>1, b_{i}(x, t) \geq 0, f(v, x, t) \geq 0$. If $\left\{b_{i}(x, t)\right\}$ is degenerate on $\Gamma_{2} \subset \partial \Omega$, then the second boundary value condition is imposed on the remaining part $\partial \Omega \backslash \Gamma_{2}$. The uniqueness of weak solution can be proved without the boundary value condition on $\Gamma_{2}$.

\section{Introduction}

The evolutionary $p(x)$-Laplacian equation,

$$
u_{t}=\operatorname{div}\left(|\nabla u|^{p(x)-2} \nabla u\right), \quad(x, t) \in Q_{T}=\Omega \times(0, T),
$$

possesses some interesting mechanical properties in the presence of an electromagnetic field [1,2], the well-posedness of weak solutions to the first initial-boundary value problem of (1) had been researched in [3-7], etc. Here, $\Omega \subset \mathbb{R}^{N}$ is a bounded domain with the smooth boundary $\partial \Omega$, and $p(x)>$ 1 is a $C^{1}(\bar{\Omega})$ function. Sobolev spaces play an important role in the theory of evolutionary $p(x)$-Laplacian equation. In recent years, the generalized Orlicz-Lebesgue spaces $L^{p}(x)$ and the corresponding generalized Orlicz-Sobolev spaces $W^{1, p(x)}$ have attracted more and more attention. The spaces $L^{p(.)}$ are special cases of the generalized Orlicz-Spaces originated by Nakano and developed by Musielak and Orlicz (see [8-10]). We refer to [11-13] for properties of the spaces $L^{p(.)}$ and $W^{k, p(.)}$ such as reflexivity, denseness of smooth functions, and Sobolev type embeddings. The study of these spaces has been stimulated by problems of elasticity, fluid dynamics, calculus of variations, and differential equations with $p(x)$-growth conditions. Roughly speaking, the interest in variable exponent spaces comes not only from their mathematical curiosity but also from their relevance in many applications such as fluid dynamics, elasticity theory, differential equations with nonstandard growth conditions, and image restoration. In addition, the study of the weak solution in other spaces such as Orlicz-Morrey space and $\dot{B}_{\infty, \infty}^{-1}$ space is a research problem (see [14-18]).

The so-called anisotropic evolutionary $\vec{p}(x)$-Laplacian equation,

$$
u_{t}=\sum_{i=1}^{N} \frac{\partial}{\partial x_{i}}\left(\left|u_{x_{i}}\right|^{p_{i}(x)-2} u_{x_{i}}\right),
$$

comes closer to the truth than equation (1) [19-21], where $\vec{p}(x)=\left(p_{1}(x), p_{2}(x), \ldots, p_{N}(x)\right)$. Recently, Zhan et al. [22$24]$ considered the first initial-boundary value problem to the equation:

$$
u_{t}=\sum_{i=1}^{N} \frac{\partial}{\partial x_{i}}\left(b_{i}(x)\left|u_{x_{i}}\right|^{p_{i}(x)-2} u_{x_{i}}\right),
$$

where $b_{i}(x) \in C^{1}(\bar{\Omega})(i=1,2, \ldots, N)$ satisfies

$$
\begin{aligned}
b_{i}(x)>0 & \text { if } x \in \Omega \\
\text { and } b_{i}(x)=0 & \text { if } x \in \partial \Omega .
\end{aligned}
$$

We have shown that this condition may act as the role of the Dirichlet boundary condition

$$
u(x, t)=0, \quad(x, t) \in \partial \Omega \times(0, T)
$$

to assure the stability of weak solutions to (3). 
In this paper, we will consider the anisotropic parabolic equation

$$
v_{t}=\sum_{i=1}^{n}\left(b_{i}(x, t)\left|v_{x_{i}}\right|^{p_{i}(x)-2} v_{x_{i}}\right)_{x_{i}}+f(v, x, t),
$$

$$
(x, t) \in Q_{T},
$$

with the initial value

$$
v(x, 0)=v_{0}(x), \quad x \in \Omega,
$$

and with a partial second boundary value condition

$$
\frac{\partial v}{\partial n}=0, \quad(x, t) \in \Gamma_{1} \times(0, T),
$$

where $b_{i}(x, t) \in C^{1}\left(\overline{Q_{T}}\right), p_{i}(x) \in C^{1}(\bar{\Omega}), p_{i}(x)>1, b_{i}(x, t) \geq$ $0, f(v(x, t), x, t) \geq 0$ and at least there is a point $x_{0} \in \Omega$ such that $f\left(v\left(x_{0}, t\right), x_{0}, t\right)>0, \Omega$ is a bounded domain with a smooth boundary $\partial \Omega$. We first assume that

$$
\begin{aligned}
\partial \Omega & =\Gamma_{1} \cup \Gamma_{2}, \\
\Gamma_{1}^{0} \cap \Gamma_{2}^{0} & =\emptyset,
\end{aligned}
$$

where $\Gamma_{1}^{0}$ and $\Gamma_{2}^{0}$ are the interior of $\Gamma_{1}$ and $\Gamma_{2}$, which are relatively open subset of $\partial \Omega$. We mainly assume that

$$
b_{i}(x, t)=0, \quad(x, t) \in \Gamma_{2} \times[0, T],
$$

denote that $p^{+}=\max _{x \in \bar{\Omega}} p(x), p^{-}=\min _{x \in \bar{\Omega}} p(x)$, for any $p(x) \in C^{1}(\bar{\Omega})$, and let

$$
\begin{aligned}
& p_{0}=\min _{x \in \bar{\Omega}}\left\{p_{1}(x), p_{2}(x), \ldots, p_{n-1}(x), p_{n}(x)\right\}, \\
& p^{0}=\max _{x \in \bar{\Omega}}\left\{p_{1}(x), p_{2}(x), \ldots, p_{n-1}(x), p_{n}(x)\right\},
\end{aligned}
$$

for any $\vec{p}(x)$. As for the anisotropic function spaces and their applications to anisotropic equations, one can refer to $[25,26]$ and the references therein.

Definition 1. If a function $v(x, t)$ satisfies that

$$
\begin{gathered}
v \in L^{\infty}\left(Q_{T}\right), \\
\frac{\partial v}{\partial t} \in L^{2}\left(Q_{T}\right), \\
b_{i}(x, t)\left|v_{x_{i}}\right|^{p_{i}(x)} \in L^{2}\left(0, T ; L^{1}(\Omega)\right),
\end{gathered}
$$

for any function $\varphi \in C^{1}\left(\overline{Q_{T}}\right), \varphi_{x_{i}} \in L^{2}\left(0, T ; L_{\text {loc }}^{p_{i}(x)}(\Omega)\right)$, $\left.\varphi\right|_{(x, t) \in \Gamma_{2} \times[0, T]}=0$,

$$
\begin{aligned}
& \iint_{\mathrm{Q}_{T}}\left[\frac{\partial v}{\partial t} \varphi+\sum_{i=1}^{n} b_{i}(x, t)\left|v_{x_{i}}\right|^{p_{i}(x)-2} v_{x_{i}} \varphi_{x_{i}}\right] d x d t \\
& \quad=\iint_{Q_{T}} f(v, x, t) \varphi d x d t,
\end{aligned}
$$

and, for any $\phi(x) \in C_{0}^{\infty}(\Omega)$,

$$
\lim _{t \rightarrow 0} \int_{\Omega} u(x, t) \phi(x) d x=\int_{\Omega} u_{0}(x) \phi(x) d x,
$$

then we say $v(x, t)$ is a weak solution of equation (6) with the initial value (7) and with the partial second value condition (8).

The main results are the following theorems.

Theorem 2. Suppose that $p_{0} \geq 2, b_{i}(x, t)$ satisfies (10) and $f, g$ are two $C^{1}$ functions satisfying

$$
f(v, s, t) \geq g(v) .
$$

Let

$$
\begin{gathered}
v_{0}(x) \in L^{\infty}(\Omega), \\
b_{i}(x, 0) v_{0 x_{i}}(x) \in L^{2}\left(0, T ; L^{p_{i}(x)}(\Omega) .\right.
\end{gathered}
$$

Then we have a positive constant $T_{1}$ such that there exists a weak solution of $(6) v(x, t) \in L^{\infty}\left(Q_{T_{1}}\right)$ with the initial condition (7) and with the partial second boundary value condition (8).

We would like to suggest that, since $f(v(x, t), x, t) \geq 0$ and at least there is a point $x_{0} \in \Omega$ such that $f\left(v\left(x_{0}, t\right), x_{0}, t\right)>0$, then the weak solution $v(x, t)$ generally blows up in a finite time [27]. However, the uniqueness of weak solution still may be true.

Theorem 3. If $b_{i}(x, t)$ satisfies (10) and $f$ is a $C^{1}$ function, and when $x$ is near to $\Gamma_{2}$,

$$
\left|b_{i}(x, t)\right| \leq c d(x)^{p_{i}^{+}-1}
$$

$v(x, t)$ and $u(x, t)$ are two solutions of (6) with the same partial second boundary value condition

$$
\frac{\partial v}{\partial n}(x, t)=\frac{\partial u}{\partial n}(x, t)=0, \quad(x, t) \in \Gamma_{1} \times(0, T),
$$

and with the same initial values

$$
v_{0}(x)=u_{0}(x), \quad x \in \Omega
$$

then

$$
v(x, t)=u(x, t), \quad(x, t) \in \Omega \times(0, T) .
$$

Here $T>T_{1}$ is the blow-up time of the weak solutions.

At the end of the introduction, we would like to suggest that it is an interesting research problem to study the anisotropic parabolic equation (6) for either $(p, q)$-Laplacian or $p$-biharmonic (see [28-30]).

\section{The Existence of Solutions}

Consider the following asymptotic problem:

$$
\begin{aligned}
v_{t}= & \sum_{i=1}^{n}\left(\left(b_{i}(x, t)+\varepsilon\right)\left(\left|v_{x_{i}}\right|^{2}+\varepsilon\right)^{\left(p_{i}(x)-2\right) / 2} v_{x_{i}}\right)_{x_{i}} \\
& +f(v, x, t), \quad(x, t) \in Q_{T},
\end{aligned}
$$


with the initial value

$$
\begin{aligned}
& v(x, 0)=v_{0 \varepsilon}(x)+\varepsilon, \quad x \in \Omega, \\
& u(x, t)=\varepsilon, \quad(x, t) \in \Gamma_{2} \times(0, T),
\end{aligned}
$$

and a partial second boundary value condition

$$
\frac{\partial v}{\partial n}=0, \quad(x, t) \in \Gamma_{1} \times(0, T),
$$

where $0<\varepsilon<1, v_{0 \varepsilon}(x) \in C_{0}^{\infty}(\Omega)$ such that

$$
\begin{aligned}
\left\|v_{0 \varepsilon}\right\|_{L^{\infty}(\Omega)} & \leq\left\|v_{0}\right\|_{L^{\infty}(\Omega)}, \\
\left\|v_{0 \varepsilon x_{i}}\right\|_{L^{p_{i}(x)}(\Omega)} & \leq\left\|v_{0 x_{i}}\right\|_{L^{p_{i}(x)}(\Omega)},
\end{aligned}
$$

and $v_{0 \varepsilon}(x)+\varepsilon \longrightarrow v_{0}(x)$ in $W^{1, \overrightarrow{p(x)}}(\Omega)$. This is possible; only we assume that $\overrightarrow{p(x)}=\left\{p_{i}(x)\right\}$ and every $p_{i}(x)$ has the logarithmic Hölder continuity [11-13]. Then similar as the usual $p$-Laplacian equation, one can show that problem (21)-(24) has a classical solution $u_{\varepsilon}$ by the classical theory for parabolic equations, and $v_{\varepsilon} \geq \varepsilon>0$ provided that $f(v(x, t), x, t) \geq 0$ and at least there is a point $x_{0} \in \Omega$ such that $f\left(v\left(x_{0}, t\right), x_{0}, t\right)>0([31]$, Theorem 4.1). We first give a lemma in a similar way as Lemma 2.1 in [32].

Lemma 4. If $f(v, x, t) \leq g(v)$ and $g(v) \in C^{1}(0, \infty)$, then there exists a $T_{1}<T$ such that

$$
\left\|v_{\varepsilon}\right\|_{L^{\infty}\left(Q_{T_{1}}\right)} \leq c\left(T_{1}\right),
$$

where $c=c\left(T_{1}\right)$ represents $c$ dependent on $T_{1}$.

Proof. Let $w(t)$ be the solution of the ordinary differential equation

$$
\begin{aligned}
\frac{d w}{d t} & =g(w), \\
w(0) & =\left\|v_{0}(x)+1\right\|_{L^{\infty}(\Omega)} .
\end{aligned}
$$

It is well known that there is a local solution $w(t), t \in\left[0, T_{0}\right] \subset$ $[0, T]$, where $T_{0}=T_{0}\left(\left\|v_{0}(x)+1\right\|_{L^{\infty}(\Omega)}\right)([33]$, Chapter 5). Let $u=v_{\varepsilon}-w$. One has

$$
\begin{gathered}
u_{t}-\sum_{i=1}^{n}\left(\left(b_{i}(x, t)+\varepsilon\right)\left(\left|(u+w)_{x_{i}}\right|^{2}+\varepsilon\right)^{\left(p_{i}(x)-2\right) / 2}\right. \\
\left.\cdot(u+w)_{x_{i}}\right)_{x_{i}}=f(v, x, t)-g(w) .
\end{gathered}
$$

Since $f(u, x, t) \geq g(u)$, one has

$$
\begin{aligned}
& f\left(v_{\varepsilon}, x, t\right)-g(w) \leq g\left(v_{\varepsilon}\right)-g(w) \\
& \quad=\left(v_{\varepsilon}-w\right) \int_{0}^{1} g^{\prime}\left(\theta v_{\varepsilon}+(1-\theta) w\right) d \theta \\
& \quad=c_{\varepsilon}(x, t)\left(v_{\varepsilon}-w\right) .
\end{aligned}
$$

From (29),

$$
\begin{gathered}
u_{t}-\sum_{i=1}^{n}\left(\left(b_{i}(x, t)+\varepsilon\right)\left(\left|(u+w)_{x_{i}}\right|^{2}+\varepsilon\right)^{\left(p_{i}(x)-2\right) / 2}\right. \\
\left.\cdot(u+w)_{x_{i}}\right)_{x_{i}}-c_{\varepsilon}(x, t) u \leq 0,
\end{gathered}
$$

with the mixed boundary condition

$$
\begin{aligned}
& u=v_{\varepsilon}-w \leq \varepsilon-\left\|u_{0}(x)+1\right\|_{L^{\infty}(\Omega)} \leq 0, \\
& \qquad(x, t) \in \Gamma_{1} \times\left[0, T_{0}\right], \\
& \frac{\partial u}{\partial n}=0, \quad(x, t) \in \Gamma_{2} \times\left[0, T_{0}\right],
\end{aligned}
$$

and

$$
\begin{aligned}
u_{0}(x)=\left[\left(v_{0 \varepsilon}(x)+\varepsilon\right)-\left\|u_{0}(x)+1\right\|_{L^{\infty}(\Omega)}\right] \leq & , \\
& x \in \bar{\Omega} .
\end{aligned}
$$

By the Hopf maximum principle, one has

$$
u(x, t) \leq 0, \quad(x, t) \in \Omega \times\left[0, T_{0}\right) .
$$

Hence, for any given $T_{1} \in\left(0, T_{0}\right)$, one has

$$
\left\|v_{\varepsilon}\right\|_{L^{\infty}\left(Q_{T_{1}}\right)} \leq c\left(T_{1}\right) \text {. }
$$
has

By multiplying (21) with $v_{\varepsilon}$ and integrating over $\Omega$, one

$$
\left\|\left(b_{i}(x, t)+\varepsilon\right) v_{\varepsilon x_{i}}\right\|_{L^{p_{i}(x)}(\Omega)} \leq c\left(T_{1}\right) .
$$

Lemma 5. If $f(v, x, t) \geq g(v)$ and $g(v) \in C^{1}(0, \infty)$, then there exists a $T_{1}<T$ such that

$$
\left\|\frac{\partial v_{\varepsilon}}{\partial t}\right\|_{L^{2}\left(Q_{T_{1}}\right)} \leq c\left(T_{1}\right) .
$$

Proof. By multiplying (21) with $\partial u_{\varepsilon} / \partial t$ and integrating over $Q_{T_{1}}$, one has

$$
\begin{aligned}
v_{\varepsilon t} & =-\sum_{i=1}^{n} \iint_{\mathrm{Q}_{T_{1}}}\left(\left(b_{i}(x, t)+\varepsilon\right)\left(\left|v_{x_{i}}\right|^{2}+\varepsilon\right)^{\left(p_{i}(x)-2\right) / 2}\right. \\
& \left.\cdot v_{x_{i}}\right)_{x_{i}} \frac{\partial v_{\varepsilon x_{i}}}{\partial t} d x d t \\
& +\iint_{\mathrm{Q}_{T_{1}}} f\left(v_{\varepsilon}, x, t\right) \frac{\partial v_{\varepsilon}}{\partial t} d x d t .
\end{aligned}
$$

Since

$$
\begin{aligned}
& \left(\left(b_{i}(x, t)+\varepsilon\right)\left(\left|v_{x_{i}}\right|^{2}+\varepsilon\right)^{\left(p_{i}(x)-2\right) / 2} v_{x_{i}}\right)_{x_{i}} \frac{\partial v_{\varepsilon x_{i}}}{\partial t} \\
& =\frac{1}{2}\left(b_{i}(x, t)+\varepsilon\right) \frac{\partial}{\partial t} \int_{0}^{\left|v_{\varepsilon x_{i}}\right|^{2}}(s+\varepsilon)^{\left(p_{i}(x)-2\right) / 2} d s
\end{aligned}
$$


accordingly

$$
\begin{aligned}
& \iint_{Q_{T_{1}}}\left(\left(b_{i}(x, t)+\varepsilon\right)\left(\left|v_{x_{i}}\right|^{2}+\varepsilon\right)^{\left(p_{i}(x)-2\right) / 2} v_{x_{i}}\right)_{x_{i}} \frac{\partial v_{\varepsilon x_{i}}}{\partial t} d x d t \\
& \quad=\frac{1}{2} \iint_{\mathrm{Q}_{T_{1}}}\left(b_{i}(x, t)+\varepsilon\right) \frac{\partial}{\partial t} \int_{0}^{\left|v_{\varepsilon x_{i}}\right|^{2}}(s+\varepsilon)^{\left(p_{i}(x)-2\right) / 2} d s d x d t \\
& \quad=\frac{1}{2} \\
& \cdot \iint_{Q_{T_{1}}} \frac{\partial}{\partial t}\left(\int_{0}^{\left|v_{\varepsilon x_{i}}\right|^{2}}\left(b_{i}(x, t)+\varepsilon\right)(s+\varepsilon)^{\left(p_{i}(x)-2\right) / 2} d s\right) d x d t \\
& \quad-\frac{1}{2} \iint_{Q_{T_{1}}} b_{i t}(x, t) \int_{0}^{\left|v_{\varepsilon x_{i}}\right|^{2}}(s+\varepsilon)^{\left(p_{i}(x)-2\right) / 2} d s d x d t=\frac{1}{2} \\
& \cdot \int_{\Omega} \int_{0}^{\left|v_{\varepsilon x_{i}}\left(x, T_{1}\right)\right|^{2}}\left(b_{i}\left(x, T_{1}\right)+\varepsilon\right)(s+\varepsilon)^{\left(p_{i}(x)-2\right) / 2} d s d x-\frac{1}{2} \\
& \cdot \int_{\Omega} \int_{0}^{\left|v_{0 \varepsilon x_{i}}\right|^{2}}\left(b_{i}(x, 0)+\varepsilon\right)(s+\varepsilon)^{\left(p_{i}(x)-2\right) / 2} d s d x-\frac{1}{2} \\
& \quad \cdot \iint_{Q_{T_{1}}} b_{i t}(x, t) \int_{0}^{\left|v_{\varepsilon x_{i}}\right|^{2}}(s+\varepsilon)^{\left(p_{i}(x)-2\right) / 2} d s d x d t .
\end{aligned}
$$

Thus by (37), using Young's inequality, one has

$$
\begin{aligned}
& \iint_{Q_{T_{1}}}\left|\frac{\partial v_{\varepsilon}}{\partial t}\right|^{2} d x d t=\sum_{i=1}^{N} \int_{\Omega} \frac{1}{p_{i}(x)}\left(b_{i}\left(x, T_{1}\right)+\varepsilon\right) \\
& \cdot\left(\left|v_{x_{i}}\left(x, T_{1}\right)\right|^{2}+\varepsilon\right)^{p_{i}(x) / 2} d x \\
& -\sum_{i=1}^{N} \int_{\Omega} \frac{1}{p_{i}(x)}\left(b_{i}(x, 0)+\varepsilon\right)\left(\left|v_{0 \varepsilon x_{i}}\right|^{2}+\varepsilon\right)^{p_{i}(x) / 2} d x \\
& -\sum_{i=1}^{N} \int_{\Omega} \frac{1}{p_{i}(x)} b_{i t}(x, t)\left(\left|v_{\varepsilon x_{i}}\right|^{2}+\varepsilon\right)^{p_{i}(x) / 2} d x \\
& +\iint_{\mathrm{Q}_{T_{1}}} f\left(v_{\varepsilon}, x, t\right) \frac{\partial v_{\varepsilon}}{\partial t} d x d t \leq c .
\end{aligned}
$$

Proof of Theorem 2. By multiplying (21) with $v_{\varepsilon}$ and integrating over $Q_{T_{1}}$, one has

$$
\begin{aligned}
& \iint_{Q_{T_{1}}} \varepsilon^{\left(p_{i}(x)-2\right) / 2}\left(b_{i}(x, t)+\varepsilon\right)\left|v_{x_{i}}\right|^{2} d x d t \leq c\left(T_{1}\right), \\
& \iint_{Q_{T_{1}}}\left(b_{i}(x, t)+\varepsilon\right)\left(\left|v_{x_{i}}\right|^{2}+\varepsilon\right)^{\left(p_{i}(x)-2\right) / 2} d x d t \\
& \quad \leq c\left(T_{1}\right)
\end{aligned}
$$

By (37), (39), (43), and (44), there is a function $v$ that satisfies

$$
\begin{gathered}
v_{\varepsilon} \longrightarrow v, \quad \text { a.e. } Q_{T_{1}}, \\
f\left(v_{\varepsilon}, x, t\right) \longrightarrow f(v, x, t), \quad \text { a.e. } Q_{T_{1}},
\end{gathered}
$$

$$
\begin{gathered}
b_{i}(x, t)^{1 / p_{i}(x)} v_{\varepsilon x_{i}} \rightarrow b_{i}(x, t)^{1 / p_{i}(x)} v_{x_{i}}, \\
\quad \text { in } L^{1}\left(0, T ; L^{p_{i}(x)}(\Omega)\right) \\
\frac{\partial v_{\varepsilon}}{\partial t} \rightarrow \frac{\partial v}{\partial t}, \quad \text { in } L^{2}\left(Q_{T_{1}}\right) .
\end{gathered}
$$

In addition, if one notices that

$$
\begin{aligned}
& \left|\left(b_{i}(x, t)+\varepsilon\right)^{\left(p_{i}(x)-1\right) / p_{i}(x)}\left(\left|v_{\varepsilon x_{i}}\right|^{2}+\varepsilon\right)\right|^{p_{i}(x) /\left(p_{i}(x)-1\right)} \\
& \quad \leq\left(b_{i}(x, t)+\varepsilon\right)\left(\left|v_{\varepsilon x_{i}}\right|^{2}+\varepsilon\right)^{p_{i}(x)\left(p_{i}(x)-2\right) / 2\left(p_{i}(x)-1\right)} \\
& \quad \cdot\left|v_{\varepsilon x_{i}}\right|^{p_{i}(x) /\left(p_{i}(x)-1\right)} \leq c\left(\left(b_{i}(x, t)+\varepsilon\right)\left|v_{\varepsilon x_{i}}\right|^{p_{i}(x)}\right. \\
& \quad+\varepsilon^{p_{i}(x)\left(p_{i}(x)-2\right) / 2\left(p_{i}(x)-1\right)}\left(b_{i}(x, t)+\varepsilon\right) \\
& \left.\quad \cdot\left|v_{\varepsilon x_{i}}\right|^{p_{i}(x) /\left(p_{i}(x)-1\right)}\right)
\end{aligned}
$$

by (43) and (44) and by that $p_{i}(x) /\left(p_{i}(x)-1\right) \leq 2$, one has

$$
\begin{aligned}
& \iint_{Q_{T_{1}}} \mid\left(b_{i}(x, t)+\varepsilon\right)^{\left(p_{i}(x)-1\right) / p_{i}(x)} \\
& \left.\cdot\left(\left|v_{\varepsilon x_{i}}\right|^{2}+\varepsilon\right)\right|^{p_{i}(x) /\left(p_{i}(x)-1\right)} d x d t \\
& \quad \leq c\left(\iint_{Q_{T_{1}}}\left(b_{i}(x, t)+\varepsilon\right)\left|v_{\varepsilon x_{i}}\right|^{p_{i}(x)} d x d t\right. \\
& +\iint_{Q_{T_{1}}}+\varepsilon^{p_{i}(x)\left(p_{i}(x)-2\right) / 2\left(p_{i}(x)-1\right)}\left(b_{i}(x, t)+\varepsilon\right) \\
& \left.\quad \cdot\left|v_{\varepsilon x_{i}}\right|^{p_{i}(x) /\left(p_{i}(x)-1\right)} d x d t\right) \\
& \quad \leq c\left(\iint_{Q_{T_{1}}}\left(b_{i}(x, t)+\varepsilon\right)\left|v_{\varepsilon x_{i}}\right|^{p_{i}(x)} d x d t\right. \\
& +c \iint_{Q_{T_{1}}}+\varepsilon^{p_{i}(x)\left(p_{i}(x)-2\right) / 2\left(p_{i}(x)-1\right)}\left(b_{i}(x, t)+\varepsilon\right) \\
& \left.\quad \cdot\left(\left|v_{\varepsilon x_{i}}\right|^{2}+1\right) d x d t\right) \leq c \iint_{\mathrm{Q}_{T_{1}}}\left(b_{i}(x, t)+\varepsilon\right) \\
& \quad \cdot\left|v_{\varepsilon x_{i}}\right|^{p_{i}(x)} d x d t+c \leq c\left(T_{1}\right) . \\
& \quad(x)
\end{aligned}
$$

Then, there exists $w_{i} \in L^{1}\left(0, T_{1} ; L^{p_{i}(x) /\left(p_{i}(x)-1\right)}(\Omega)\right), i=$ $1,2, \ldots, n$ such that

$$
\begin{array}{r}
\left(b_{i}(x, t)+\varepsilon\right)^{\left(p_{i}(x)-1\right) / p_{i}(x)}\left(\left|v_{\varepsilon x_{i}}\right|^{2}+\varepsilon\right)^{\left(p_{i}(x)-2\right) / 2} \rightarrow w_{i}, \\
\quad \operatorname{in~} L^{1}\left(0, T_{1} ; L^{p_{i}(x) /\left(p_{i}(x)-1\right)}(\Omega)\right) .
\end{array}
$$


Using the similar method as that of the evolutionary $p$-Laplacian equation [34], we can deduce that

$$
\begin{aligned}
& \lim _{\varepsilon \longrightarrow 0} \iint_{Q_{T}} \sum_{i=1}^{N}\left|v_{\varepsilon x_{i}}\right|^{p_{i}(x)-2} v_{\varepsilon x_{i}} \xi_{x} d x d t \\
& \quad=\iint_{Q_{T}} \sum_{i=1}^{N} w_{i} \xi_{x_{i}} d x d t \\
& \quad=\iint_{Q_{T}} \sum_{i=1}^{N}\left|v_{x_{i}}\right|^{p_{i}(x)-2} v_{x_{i}} \xi_{x_{i}} d x d t,
\end{aligned}
$$

for any $\xi(x, t) \in C_{0}^{1}\left(Q_{T}\right)$.

At last, the initial value in the sense of (14) can be found in [3]. Consequently, $v_{\varepsilon}$ is the solution of (6).

\section{The Stability}

One can refer to [11-13] for the definitions of the exponent variable spaces, $\left(L^{q(x)}(\Omega),|\cdot|_{L^{q(x)}(\Omega)}\right),\left(W^{1, q(x)}(\Omega),|\cdot|_{W^{1, q(x)}(\Omega)}\right)$, and $W_{0}^{1, q(x)}(\Omega)$. Also, one can find other details and recent applications to partial differential equations in [35-39].

Lemma 6 (see [11-13]). If $p(x)$ and $q(x)$ are real functions with $1 / p(x)+1 / q(x)=1$ and $q(x)>1$, then, for any $v \in L^{p(x)}(\Omega)$ and $u \in L^{q(x)}(\Omega)$, we have

$$
\left|\int_{\Omega} v u d x\right| \leq 2|v|_{L^{p(x)}(\Omega)}|u|_{L^{q(x)(\Omega)}} .
$$

\section{Moreover,}

$$
\text { if }|v|_{L^{q(x)}(\Omega)}=1 \text {, then } \int_{\Omega}|v|^{q(x)} d x=1,
$$$$
\text { if }|v|_{L^{q(x)}}(\Omega)>1 \text {, }
$$

$$
\text { then }|v|_{L^{q(x)}}^{q^{-}} \leq \int_{\Omega}|v|^{q(x)} d x \leq|v|_{L^{q(x)}}^{q^{+}},
$$

if $|v|_{L^{q(x)}}(\Omega)<1$,

$$
\text { then }|v|_{L^{q(x)}}^{q^{+}} \leq \int_{\Omega}|v|^{q(x)} d x \leq|v|_{L^{q(x)}}^{q^{-}} .
$$

We let $g_{m}(s)$ be an odd function, and

$$
g_{m}(s)= \begin{cases}1, & s>\frac{1}{m}, \\ m^{2} s^{2} \mathrm{e}^{1-m^{2} s^{2}}, & 0 \leq s \leq \frac{1}{m} .\end{cases}
$$

Then,

$$
\lim _{m \longrightarrow \infty} g_{m}(s)=\operatorname{sgn}(s), \quad s \in(-\infty,+\infty)
$$

Let $\varphi(x)$ be a $C^{1}(\bar{\Omega})$ function satisfying

$$
\begin{gathered}
\left.\varphi(x)\right|_{x \in \Gamma_{2}}=0, \\
\left.\varphi(x)\right|_{x \in \bar{\Omega} \backslash \Gamma_{2}}>0,
\end{gathered}
$$

and

$$
\Omega_{m}=\left\{x \in \Omega: \varphi(x)>\frac{1}{m}\right\} .
$$

Define

$$
\varphi_{m}(x)= \begin{cases}1, & \text { if } x \in \Omega_{m} \\ m \varphi(x), & \text { if } x \in \Omega \backslash \Omega_{m}\end{cases}
$$

Then $\left.\varphi_{m}(x)\right|_{x \in \Gamma_{2}}=0$ and

$$
\varphi_{m x_{i}}(x)= \begin{cases}0, & \text { if } x \in \Omega_{m}, \\ m \varphi_{x_{i}}(x), & \text { if } x \in \Omega \backslash \Omega_{m} .\end{cases}
$$

Theorem 7. Let $v(x, t) \in L^{\infty}\left(Q_{T}\right)$ and $u(x, t) \in L^{\infty}\left(Q_{T}\right)$ be two solutions of (6) with the same partial second boundary value condition (8) and with the initial values $v_{0}(x)$ and $u_{0}(x)$. If $\partial \Omega=\Gamma_{1} \cup \Gamma_{2}$ satisfies (9), $b_{i}$ satisfies (10),

$$
|f(v, x, t)-f(u, x, t)| \leq c|v-u|
$$

and, for large enough $m$,

$$
m e s s \sup _{t \in[0, T]}\left(\int_{\Omega \backslash \Omega_{m}} b_{i}(x, t)\left|\varphi(x)_{x_{i}}\right|^{p_{i}(x)} d x\right)^{1 / p_{i}^{+}} \leq c ;
$$

then

$$
\int_{\Omega}|v(x, t)-u(x, t)| d x \leq \int_{\Omega}|v(x, 0)-u(x, 0)| d x,
$$

$$
\text { a.e. } t \in[0, T] \text {. }
$$

Proof. Let $\chi_{[\tau, s]}$ be the characteristic function of $[\tau, s) \subseteq$ $[0, T)$. By a process of limit, we can choose the test function as $\chi_{[\tau, s]} \varphi_{m} g_{m}(v-u)$. Then

$$
\begin{gathered}
\int_{\tau}^{s} \int_{\Omega} \varphi_{m} g_{m}(v-u) \frac{\partial(v-u)}{\partial t} d x d t+\sum_{i=1}^{n} \int_{\tau}^{s} \int_{\Omega} b_{i}(x) \\
\cdot\left(\left|v_{x_{i}}\right|^{p_{i}(x)-2} v_{x_{i}}-\left|u_{x_{i}}\right|^{p_{i}(x)-2} u_{x_{i}}\right)\left(v_{x_{i}}-u_{x_{i}}\right) \\
\cdot g_{m}^{\prime}(v-u) \varphi_{m}(x) d x d t+\sum_{i=1}^{n} \int_{\tau}^{s} \int_{\Omega} b_{i}(x) \\
\cdot\left(\left|v_{x_{i}}\right|^{p_{i}(x)-2} v_{x_{i}}-\left|u_{x_{i}}\right|^{p_{i}(x)-2} u_{x_{i}}\right)\left(v_{x_{i}}-u_{x_{i}}\right) \\
\cdot g_{m}(v-u) \varphi_{m x_{i}} d x d t=\int_{\tau}^{s} \int_{\Omega}[f(v, x, t) \\
-f(u, x, t)] \varphi_{m} g_{m}(v-u) d x d t .
\end{gathered}
$$

First of all,

$$
\begin{gathered}
\int_{\tau}^{s} \int_{\Omega} b_{i}(x)\left(\left|v_{x_{i}}\right|^{p_{i}(x)-2} v_{x_{i}}-\left|u_{x_{i}}\right|^{p_{i}(x)-2} u_{x_{i}}\right) \\
\cdot\left(v_{x_{i}}-u_{x_{i}}\right) g_{m}^{\prime}(v-u) \varphi_{m}(x) d x d t \geq 0 .
\end{gathered}
$$


Secondly, since $v_{t} \in L^{2}\left(Q_{T}\right), u_{t} \in L^{2}\left(Q_{T}\right)$, by the Lebesgue dominated convergence theorem, we have

$$
\begin{array}{r}
\lim _{m \rightarrow \infty} \int_{\tau}^{s} \int_{\Omega} \varphi_{m}(x) g_{m}(v-u) \frac{\partial(v-u)}{\partial t} d x d t \\
=\int_{\Omega}|v-u|(x, s) d x-\int_{\Omega}|v-u|(x, \tau) d x .
\end{array}
$$

By (59), we have

$$
\begin{aligned}
& \mid \int_{\tau}^{s} \int_{\Omega} b_{i}(x, t)\left(\left|v_{x_{i}}\right|^{p_{i}(x)-2} v_{x_{i}}-\left|u_{x_{i}}\right|^{p_{i}(x)-2} u_{x_{i}}\right) \\
& \text { - } \varphi_{m x_{i}} g_{m}(v-u) d x d t|=| \int_{\tau}^{s} \int_{\Omega \backslash \Omega_{m}} b_{i}(x, t) \\
& \cdot\left(\left|v_{x_{i}}\right|^{p_{i}(x)-2} v_{x_{i}}-\left|u_{x_{i}}\right|^{p_{i}(x)-2} u_{x_{i}}\right) \\
& \text { - } \varphi_{m x_{i}} g_{m}(v-u) d x d t \mid \leq m \int_{\tau}^{s} \int_{\Omega \backslash \Omega_{m}} b_{i}(x, t) \\
& \cdot\left(\left|v_{x_{i}}\right|^{p_{i}(x)-1}+\left|u_{x_{i}}\right|^{p_{i}(x)-1}\right) \\
& \cdot\left|\varphi_{x_{i}} g_{m}(v-u)\right| d x d t \\
& \leq c m \int_{\tau}^{s}\left(\int_{\Omega \backslash \Omega_{m}} b_{i}(x, t)\right. \\
& \left.\cdot\left(\left|v_{x_{i}}\right|^{p_{i}(x)}+\left|u_{x_{i}}\right|^{p_{i}(x)}\right) d x\right)^{1 / q_{i}^{+}}\left(\int_{\Omega \backslash \Omega_{m}} b_{i}(x, t)\right. \\
& \left.\cdot\left|\varphi_{x_{i}}\right|^{p_{i}(x)} d x\right)^{1 / p_{i}^{+}} d t \\
& \leq c \int_{\tau}^{s}\left[\left(\int_{\Omega \backslash \Omega_{m}} b_{i}(x, t)\left|v_{x_{i}}\right|^{p_{i}(x)} d x\right)^{1 / q_{i}^{+}}\right. \\
& \left.+\left(\int_{\Omega \backslash \Omega_{m}} b_{i}(x, t)\left|u_{x_{i}}\right|^{p_{i}(x)} d x\right)^{1 / q_{i}^{+}}\right] \\
& \cdot m\left(\int_{\Omega \backslash \Omega_{m}} b_{i}(x, t)\left|\varphi_{x_{i}}\right|^{p_{i}(x)} d x\right)^{1 / p_{i}^{+}} d t \\
& \leq c \int_{\tau}^{s}\left(\int_{\Omega \backslash \Omega_{m}} b_{i}(x, t)\left|v_{x_{i}}\right|^{p_{i}(x)} d x\right)^{1 / q_{i}^{+}} d t \\
& +c \int_{\tau}^{s}\left(\int_{\Omega \backslash \Omega_{m}} b_{i}(x, t)\left|u_{x_{i}}\right|^{p_{i}(x)} d x\right)^{1 / q_{i}^{+}} d t,
\end{aligned}
$$

where $q_{i}(x)=p_{i}(x) /\left(p_{i}(x)-1\right), q_{i}^{+}=\max _{x \in \bar{\Omega}} q_{i}(x)$.

Then we have

$$
\begin{aligned}
& \lim _{m \rightarrow \infty} \mid \int_{\tau}^{s} \int_{\Omega} b_{i}(x, t)\left(\left|v_{x_{i}}\right|^{p_{i}(x)-2} v_{x_{i}}-\left|u_{x_{i}}\right|^{p_{i}(x)-2} u_{x_{i}}\right) \\
& \cdot \varphi_{m x_{i}} g_{m}(v-u) d x d t \mid
\end{aligned}
$$

$$
\begin{aligned}
& \leq c \lim _{m \longrightarrow \infty}\left[\left(\int_{\Omega \backslash \Omega_{m}} b_{i}(x, t)\left|v_{x_{i}}\right|^{p_{i}(x)} d x\right)^{1 / q_{i}^{+}}\right. \\
& \left.+\left(\int_{\Omega \backslash \Omega_{m}} b_{i}(x, t)\left|u_{x_{i}}\right|^{p_{i}(x)} d x\right)^{1 / q_{i}^{+}}\right]=0 .
\end{aligned}
$$

In addition, by (58)

$$
\begin{aligned}
& \lim _{m \rightarrow \infty} \mid \int_{\tau}^{s} \int_{\Omega}[f(v, x, t)-f(u, x, t)] \\
& \cdot \varphi_{m} g_{m}(v-u) d x d t\left|\leq \int_{\tau}^{s} \int_{\Omega}\right| v(x, t) \\
& -u(x, t) \mid d x d t .
\end{aligned}
$$

At last, let $m \longrightarrow \infty$ in (61). Then

$\int_{\Omega}|v(x, s)-u(x, s)| d x \leqslant \int_{\Omega}|v(x, \tau)-u(x, \tau)| d x$.

By the arbitrary of $\tau$, we have the conclusion.

Proof of Theorem 3. We only need to choose

$$
\varphi(x)=d(x)
$$

in Theorem 7, the conclusion is clear.

\section{Conclusion}

Comparing with the isotropic case, the anisotropic parabolic equations are closer to the truth about the applications. They reflect in the mathematical modeling of physical and mechanical processes in anisotropic continuous medium. Different from the elliptic anisotropic equations with the variable exponent that has attracted much attentions recently, more or less beyond my imagination, only a few references related to the anisotropic parabolic equations can be found. Even there are not any papers to discuss the fundamental solution of the anisotropic equation. This paper has taken this one step further. We think the contributions are mainly in two aspects. The first one lies in the fact that if the diffusion coefficient $b_{i}(x, t)$ is degenerate on a part of the boundary $\Gamma_{1} \subset \partial \Omega$, then, only under the partial second boundary value condition, one can study the well-posedness of solutions to the anisotropic parabolic equations. The second one lies in the fact that even if the weak solutions may be blow-up in finite time provided that the source $f(x, t, u) \geq 0$, the uniqueness of weak solution still may be true.

\section{Data Availability}

There is not any data in the paper.

\section{Conflicts of Interest}

The author declares that he has no conflicts of interest. 


\section{Acknowledgments}

The paper is supported by NSF of Fujian Province and SF of Xiamen University of Technology, China.

\section{References}

[1] M. Ružička, Electrorheological Fluids: Modeling and Mathematical Theory, vol. 1748 of Lecture Notes in Mathematics, Springer, Berlin, Germany, 2000.

[2] E. Acerbi and G. Mingione, "Regularity results for stationary electro-rheological fluids," Archive for Rational Mechanics and Analysis, vol. 164, no. 3, pp. 213-259, 2002.

[3] S. Antontsev and S. Shmarev, "Anisotropic parabolic equations with variable nonlinearity," Publicacions Matemàtiques, vol. 53, no. 2, pp. 355-399, 2009.

[4] S. Antontsev and S. Shmarev, "Parabolic equations with double variable nonlinearities," Mathematics and Computers in Simulation, vol. 81, no. 10, pp. 2018-2032, 2011.

[5] S. Lian, W. Gao, H. Yuan, and C. Cao, "Existence of solutions to an initial Dirichlet problem of evolutional $\mathrm{p}(\mathrm{x})$-Laplace equations," Annales de l'Institut Henri Poincaré C, Analyse Non Linéaire, vol. 29, no. 3, pp. 377-399, 2012.

[6] G. Akagi and K. Matsuura, "Nonlinear diffusion equations driven by the p(.)-Laplacian," Nonlinear Differential Equations and Applications, vol. 20, no. 1, pp. 37-64, 2013.

[7] B. Guo and W. Gao, "Finite-time blow-up and extinction rates of solutions to an initial Neumann problem involving the $\mathrm{p}(\mathrm{x}, \mathrm{t})$-Laplace operator and a non-local term," Discrete and Continuous Dynamical Systems, vol. 36, no. 2, pp. 715-730, 2016.

[8] J. Musielak, Orlicz Spaces and Modular Spaces, Springer-Verlag, Berlin, Germany, 1983.

[9] J. Musielak and W. Orlicz, "On modular spaces," Studia Mathematica, vol. 18, pp. 591-597, 1959.

[10] H. Nakano, Modular Semi-Ordered Spaces, Maruzen Co. Ltd., Tokyo, Japan, 1950.

[11] V. V. Zhikov, "On the density of smooth functions in SobolevOrlicz spaces," Journal of Mathematical Sciences, vol. 132, no. 3, pp. 285-294, 2006.

[12] X. L. Fan and D. Zhao, "On the spaces $L^{p(x)(\Omega)}$ and $W^{m, p(x), " ~}$ Journal of Mathematical Analysis and Applications, vol. 263, no. 2, pp. 424-446, 2001.

[13] O. Kovácik and J. Rákosnk, "On spaces $L^{p(x)}$ and $W^{1, p(x)}$," Czechoslovak Mathematical Journal, vol. 41, no. 116, pp. 592-618, 1991.

[14] S. Gala, Q. Liu, and M. A. Ragusa, "A new regularity criterion for the nematic liquid crystal flows," Applicable Analysis: An International Journal, vol. 91, no. 9, pp. 1741-1747, 2012.

[15] S. Gala, Q. Liu, and M. A. Ragusa, "Logarithmically improved regularity criterion for the nematic liquid crystal flows in Bळ, $\infty-1$ space," Computers and Mathematics with Applications, vol. 65, no. 11, pp. 1738-1745, 2013.

[16] S. Gala and M. A. Ragusa, "A logarithmically improved regularity criterion for the 3D MHD equations in Morrey-Campanato space," Applicable Analysis: An International Journal, vol. 2, no. 1, pp. 16-23, 2017.

[17] F. Deringoz, V. S. Guliyev, and M. A. Ragusa, "Intrinsic square functions on vanishing generalized Orlicz-Morrey spaces," SetValued and Variational Analysis, vol. 25, no. 4, pp. 807-828, 2017.

[18] S. Gala, Z. Guo, and M. A. Ragusa, "A remark on the regularity criterion of Boussinesq equations with zero heat conductivity," Applied Mathematics Letters, vol. 27, pp. 70-73, 2014.
[19] A. S. Tersenov, "The one dimensional parabolic p(x)-Laplace equation," Nonlinear Differential Equations and Applications, vol. 23, no. 3, pp. 1-11, 2016.

[20] A. S. Tersenov and A. S. Tersenov, "Existence of lipschitz continuous solutions to the Cauchy-Dirichlet problem for anisotropic parabolic equations," Journal of Functional Analysis, vol. 272, no. 10, pp. 3965-3986, 2017.

[21] J. Aramaki, "Hölder continuity with exponent $(1+\alpha) / 2$ in the time variable for solutions of parabolic equations," Electronic Journal of Differential Equations, vol. 96, pp. 1-6, 2015.

[22] H. Zhan, "The stability of the anisotropic parabolic equation with the variable exponent," Boundary Value Problems, vol. 2017, no. 134, pp. 1-14, 2017.

[23] H. Zhan and J. Wen, "Evolutionary $\mathrm{p}(\mathrm{x})$-Laplacian equation free from the limitation of the boundary value," Electronic Journal of Differential Equations, vol. 143, pp. 1-13, 2016.

[24] H. Zhan and Z. Feng, "Solutions of evolutionary p(x)-Laplacian equation based on the weighted variable exponent space," Zeitschrift für angewandte Mathematik und Physik, vol. 68, no. 134, pp. 1-17, 2017.

[25] M. A. Ragusa and A. Scapellato, "Mixed Morrey spaces and their applications to partial differential equations," Nonlinear Analysis. Theory, Methods \& Applications. An International Multidisciplinary Journal, vol. 151, pp. 51-65, 2017.

[26] A. Scapellato, "New perspectives in the theory of some function spaces and their applications," AIP Conference Proceedings 1978, vol. 1978, no. 1, Article ID 140002, 2018.

[27] S. Antontsev and S. Shmarev, "Blow-up of solutions to parabolic equations with nonstandard growth conditions," Journal of Computational and Applied Mathematics, vol. 234, no. 9, pp. 2633-2645, 2010.

[28] S. M. Khalkhali and A. Razani, "Multiple solutions for a quasilinear (p,q)-elliptic system," Electronic Journal of Differential Equations, vol. 144, pp. 1-14, 2013.

[29] F. Behboudi and A. Razani, "Two weak solutions for a singular (p,q)-Laplacian problem,” Filomat, 2019.

[30] M. Makvand Chaharlang and A. Razani, "A fourth order singular elliptic problem involving p-biharmonic operator," Taiwanese Journal of Mathematics, 2019.

[31] O. A. Ladyzenskaja, V. A. Solonnikov, and N. N. Uralceva, Linear and Quasilinear Equations of Parabolic Type, vol. 23, Translations of Mathematical Monographs, American Mathematical Society, Providence, RI, USA, 1968.

[32] J. Wang, S. Chong, and W. Gao, "Eixstence of local solutions to a class of doubly degenerate parabolic equations," Northeastern Mathematical Journal, vol. 23, no. 2, pp. 157-166, 2007.

[33] C. K. Zhong, X. L. Fan, and W. Y. Chen, Nonlinear, Functional Analysis, Lanzhou University Press, Lanzhou, China, 1998.

[34] Z. Q. Wu, J. N. Zhao, J. X. Yin, and H. Li, Nonlinear Diffusion Equations, World Scientific Publishing, Singapore, 2001.

[35] R. Mahdavi Khanghahi and A. Razani, "Solutions for a singular elliptic problem involving the $\mathrm{p}(\mathrm{x})$-laplacian," Filomat, vol. 32, no. 14, pp. 4841-4850, 2018.

[36] M. Makvand Chaharlang and A. Razani, "Existence of infinitely many solutions for a class of nonlocal problems with Dirichlet boundary condition," Communications of the Korean Mathematical Society, vol. 34, no. 1, pp. 155-167, 2019.

[37] M. Makvand Chaharlang and A. Razani, "Infinitely many solutions for a fourth order singular elliptic problem," Filomat, vol. 32, no. 14, pp. 5003-5010, 2018. 
[38] A. Scapellato, "Homogeneous Herz spaces with variable exponents and regularity results," Electronic Journal of Qualitative Theory of Differential Equations, vol. 82, pp. 1-11, 2018.

[39] A. Scapellato, "Regularity of solutions to elliptic equations on Herz spaces with variable exponents," Boundary Value Problems, vol. 2019, no. 2, 2019. 


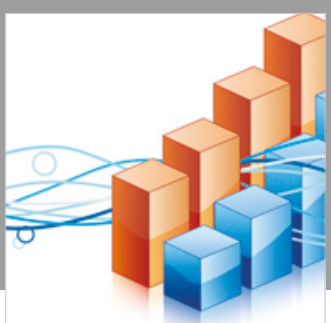

Advances in

Operations Research

\section{-n-m}
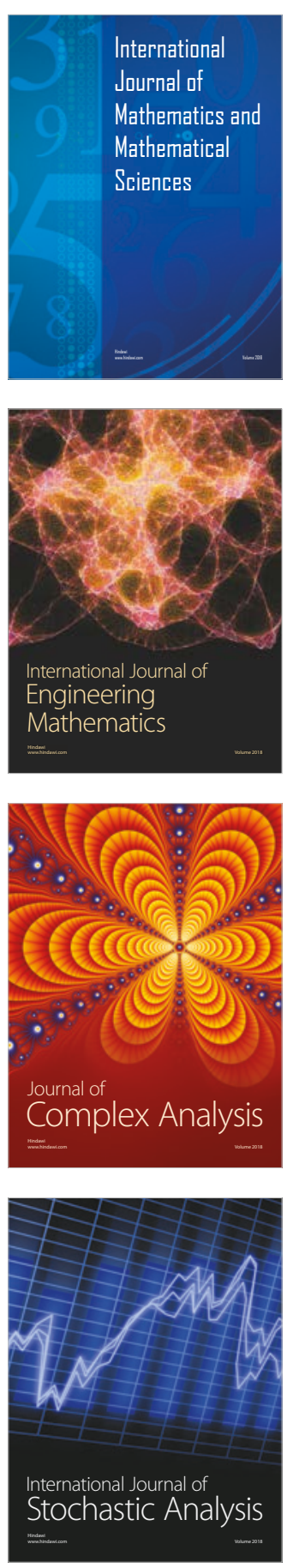
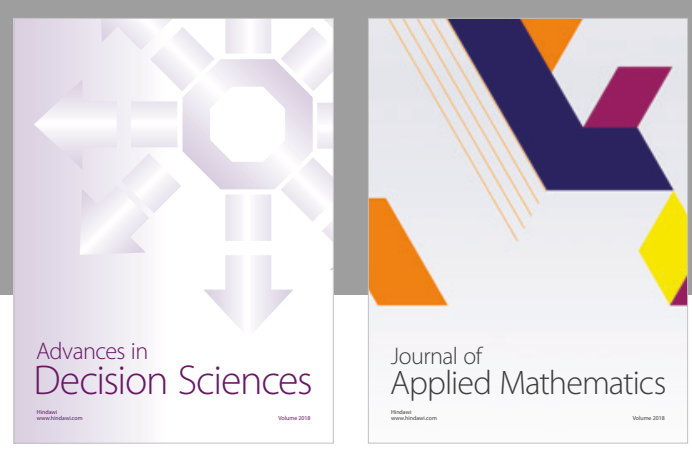

Journal of

Applied Mathematics
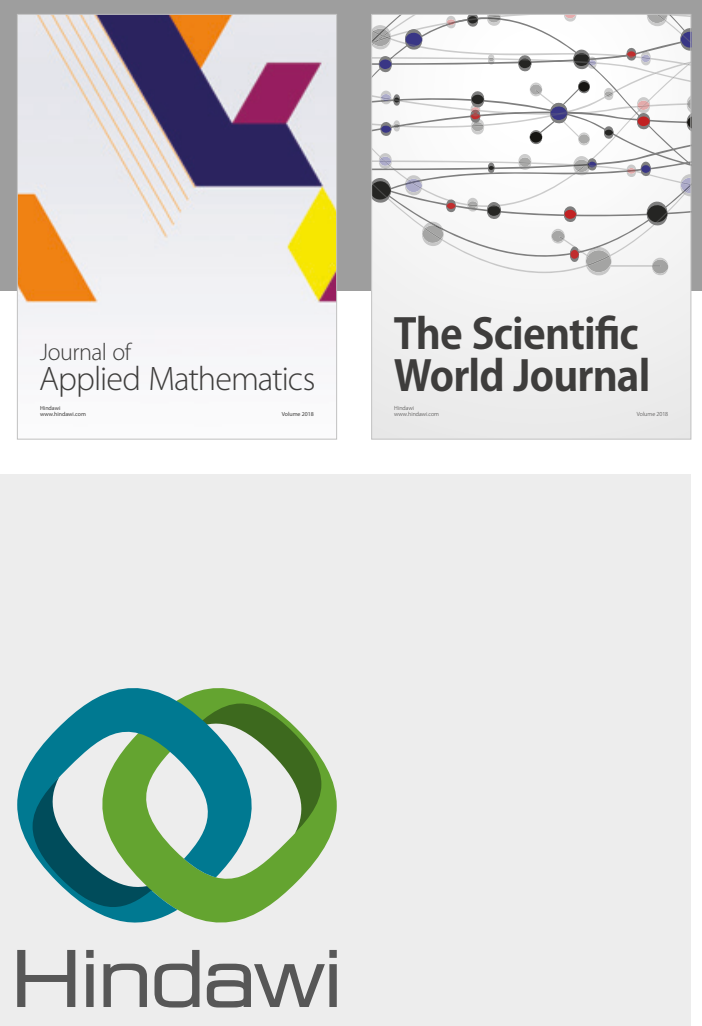

Submit your manuscripts at

www.hindawi.com

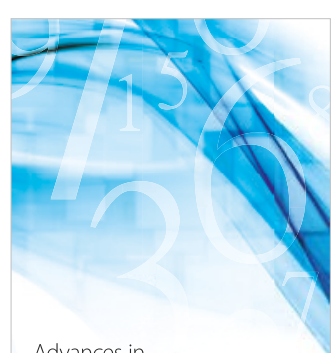

Advances in
Numerical Analysis
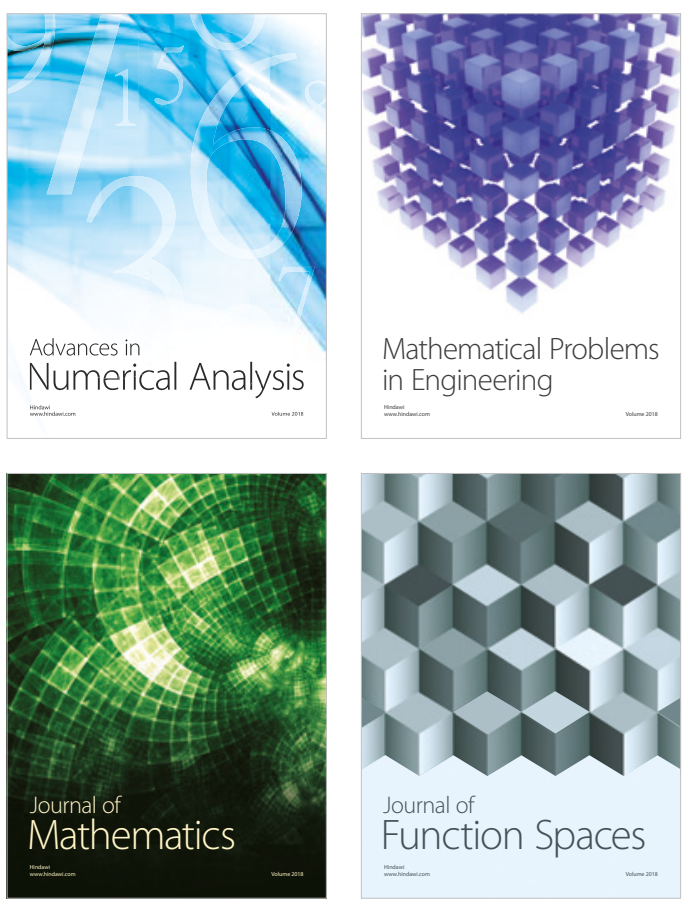

Mathematical Problems in Engineering

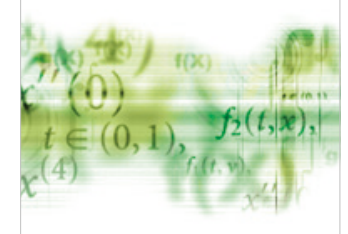

International Journal of

Differential Equations

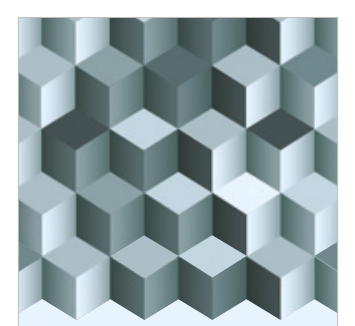

Journal of

Function Spaces

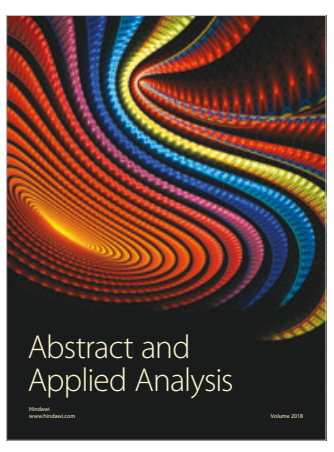

The Scientific

World Journal

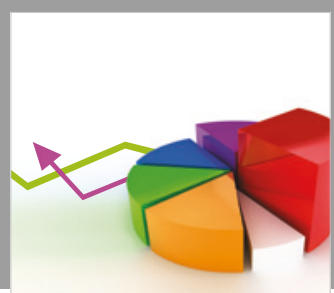

Journal of

Probability and Statistics
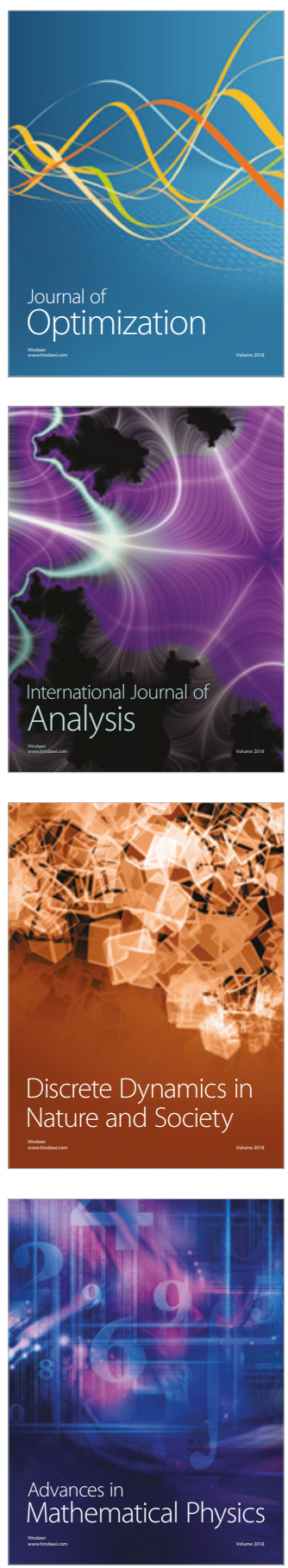